\title{
Cartographie De L'évolution Démographique Et Atteinte De L’objectif Du Développement Durable Dans Le Secteur De L'eau Dans Le Hollidjé Au Sud- Est Du Bénin
}

\author{
Rachade O.A. Djinadou \\ Léon Baní Bio Bigou \\ Azaria Oladjide \\ Armand Vodounou
}

Laboratoire d'Etude de la Dynamique Urbaine et Régionale

UAC : Université d’Abomey-Calavi (Bénin)

doi: 10.19044/esj.2017.v13n14p110 URL:http://dx.doi.org/10.19044/esj.2017.v13n14p110

\begin{abstract}
At the end of the Millennium Development Goals in 2015, the issue of access to water has not been fully addressed in developing countries in the face of increasing population. In Benin, despite the efforts made in this sector, rural populations are still faced with difficulties in accessing drinking water. This study aims to contribute to a better cartographic knowledge of the demographic evolution and its implications on the access to drinking water in Hollidjé. The study was carried out in 33 Holli villages in the municipalities of Pobè and Adja-Ouèrè. The censuses of 1979-1992-2002 and 2013 and the base of the water points of the Directorate General of Water of 2015 were used. These data have been processed using SPSS software 23, DEMProj for demographic projection by 2030 and ArcGis 10.1 for data spatialization and mapping. The study shows that Hollidjé is growing at a rate of $6.6 \%$ a year, double the national increase (3.50\%). With a density of more than $200 \mathrm{hbts} / \mathrm{km}^{2}$, this territory has a low drinking water supply rate (minus $40 \%$ in 2013). By 2030, the Hollidjè population will double. Thus, the equivalent drinking water requirement will be 500 water points. At this pace, the objectives of sustainable development related to drinking water and sanitation may not be achieved if sectoral policies are not implemented.
\end{abstract}

Keywords: Benin, Pobè, Hollidjè, Cartography, population growth; Access to drinking water; SDG 


\section{Résumé}

Au terme des Objectifs du Millénaire pour le Développement en 2015, la question de l'accès à l'eau n’a pas été totalement réglée dans les pays en développement face à l’accroissement démographique. Au Bénin, malgré les efforts réalisés dans ce secteur, des populations rurales sont toujours confrontées aux difficultés d'accès à l'eau potable. Cette étude vise à contribuer à une meilleure connaissance cartographique de l'évolution démographique et de ses implications sur l'accès à l'eau potable dans le Hollidjé. L’étude a été menée dans 33 villages Holli dans les communes de Pobè et Adja-Ouèrè. Les recensements de 1979-1992-2002 et 2013 et la base des points d'eau de la Direction Générale de l'Eau de 2015 ont été utilisées. Ces données ont été traitées avec les logiciels SPSS 23, DEMProj pour la projection démographique à l'horizon 2030 et ArcGis 10.1 pour la spatialisation des données et l'élaboration des figures cartographiques. Il ressort de cette étude que le Hollidjé connaît un accroissement rapide de sa population de l'ordre de 6,6 \% l'an, soit le double de l'accroissement national (3,50\%). Avec une densité de plus de $200 \mathrm{hbts} / \mathrm{km}^{2}$, ce territoire a un faible taux de desserte en eau potable (moins 40\% en 2013). A l'horizon 2030, la population du Hollidjè va doubler. Ainsi, les besoins en eau potable équivalent seront de 500 points d'eau. A cette allure, les objectifs du développement durable liés à l'eau potable et à l'assainissement risquent de ne pas être atteints si des politiques sectorielles ne sont pas mises en œuvre.

Mots clés : Bénin, Pobè, Hollidjè, Cartographie, croissance démographie ; Accès à l'eau potable ; ODD

\section{Introduction}

Avec la révolution industrielle et notamment les progrès dans le domaine de la médecine, le monde a connu une augmentation rapide de sa population. En effet, la population mondiale augmente actuellement d'un peu plus de 200000 habitants par jour, soit près de 80 millions par an (FUNAP, 2014). Selon cette même source, la population mondiale dépasserait le cap de 9 milliards en 2045. Les pays de l'Afrique et notamment ceux de l'Afrique subsaharienne ne sont pas restés en marge de cette croissance démographique. En 2007, l'Afrique subsaharienne abritait 807 millions d'habitants, soit 84 \% de la population du continent africain; la progression africaine est donc essentiellement liée à celle de l'Afrique subsaharienne (Djinadou, 2012). Ainsi, le Bénin, à l'instar des autres pays de la sousrégion, connait également une augmentation rapide de sa population. En 2013, le quatrième recensement de la population a dénombré 10008749 habitants contre 6769914 habitants au recensement de 2002, soit un taux annuel d'accroissement intercensitaire de 3,5\% (INSAE/RGPH4, 2013). 
Cette croissance démographique rapide que connait le monde a permis à l'eau d'acquérir une valeur dont le cours est indexé sur la pénurie annoncée et lié à la consommation individuelle en pleine explosion (Niang, 2008). À partir de 1950, les niveaux de consommation en eau ont été multipliés par six (FUNAP, 2014). Cette augmentation des besoins a entrainé l'inégale répartition des ressources en eau et de ses dérivées, d'où le déficit criard dans de nombreuses régions du monde.

En 2010, 2,6 milliards de personnes n’ont pas accès à des infrastructures d'assainissement qui garantissent une protection minimale de l'eau consommée dans la suite du cycle par la population, soit un taux de 39 $\%$ de la population mondiale et 4 milliards de personnes soit $61 \%$ de la population mondiale n'ont pas accès à une meilleure hygiène. (UNICEF, 2015/http://www.wsp.org/). En 2030, la population mondiale serait estimée à environ 8 milliards de personnes; ce qui pourrait aggraver la rareté des ressources et entrainer une pénurie progressive d'eau. Ceci est d'autant plus inquiétant car la demande en eau est supérieure de 56\% aux réserves. En Afrique, d'ici 2025, 600 millions d'individus n'auront pas accès à l'eau potable (Niang, 2008).

Au Benin, en dépit des efforts fournis, le taux de desserte en eau potable en milieu rural n'était que $42 \%$ en 2010 . Autrement dit, près de $60 \%$ de la population rurale n'ont pas accès à l'eau potable et aux infrastructures d'assainissement. Dans cet ensemble, le département du Plateau affiche un niveau bas par rapport au niveau national avec 32\% de couverture en eau potable (OMS-UNICEF, 2010). Cette situation est plus préoccupante en milieu rural et précisément en milieu Holli où les caractéristiques géopédologiques ne sont pas du tout favorable à l'installation des infrastructures d'approvisionnement en eau potable et assainissement (Azontonde, 1991 et Lanokou, 2016). Malgré les contraintes de la nature du milieu Holli, les populations s'y installent à cause des caractéristiques pédologiques très favorables pour la production agricole et l'exploitation du sol. Il est alors urgent de chercher à savoir si les objectifs du développement durable seront atteints dans cette région.

\section{Matériel et Méthode}

La réalisation de cette étude a nécessité la collecte des données démographiques issues des quatre recensements (1979, 1992, 2002 et 2013) ainsi que la base des points d'eau de 2015 du département du Plateau fournies par la Direction Générale de l'Eau. La cartographie des données démographiques des différentes années a nécessité la création des bases de données démographiques géo-référencée pour chaque année qui ont ensuite été spatialisées sur le fond topographique de la zone d'étude préalablement 
extraite grâce au logiciel ArcGis 10.1. Le traitement de ces données a abouti à l'élaboration de diverses cartes thématiques.

Le découpage en classe de ces données en vue de l’obtention de classes d'amplitudes égales a été réalisé par la méthode de discrétisation comme le montre la figure 1

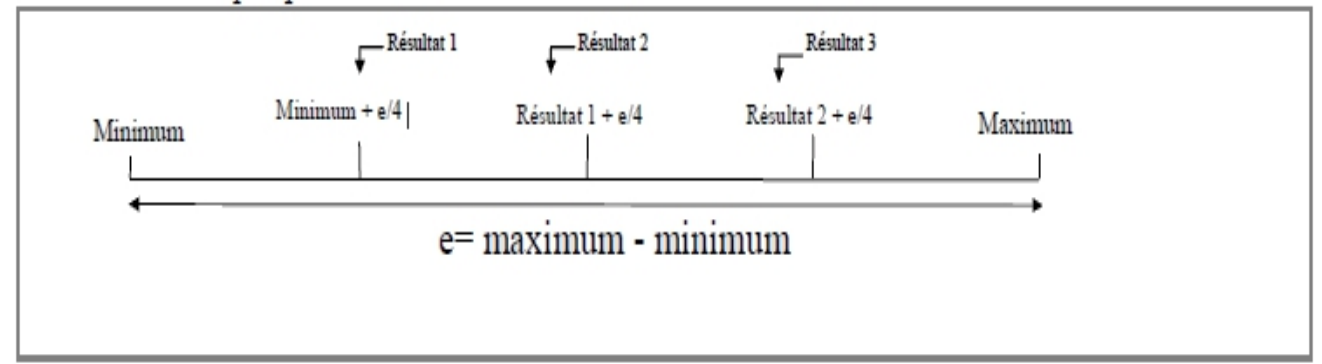

Figure 1 : Amplitudes des classes

La population cible de cette étude est composée des peuples Hollis qui vivent dans la zone traversée par la dépression médiane dans le département du plateau (Pobè et Adja-Ouèrè). Avec 33 villages et 10803 ménages.

Les estimations de la population et des besoins en eau ont été faites sur 15 ans c'est-à-dire à l'horizon 2030. Cette projection démographique s'est faite à partir de la formule suivante en tenant compte du scénario simple: $\mathbf{P}_{\mathbf{1}}=\mathbf{P}_{\mathbf{0}}(\mathbf{1}+\mathbf{r})^{\mathbf{t}}$ avec $\mathbf{P}_{\mathbf{1}}$ : Population projetée (2030), $\mathbf{P}_{\mathbf{0}}$ : Population initiale (2015) dont la base est le recensement de 2013; r: Taux d'accroissement moyen annuel et $\mathbf{t}$ : Nombre d'années (15 ans).

L'estimation des besoins en point d'eau dans le Hollidjé s'est faite en considérant la norme d'équipement en point d'eau de la Direction Générale de l'Eau du Bénin qui est de 250 habitants pour l'installation d'un point d'eau.

\footnotetext{
Taux de desserte $=\frac{\text { Population desservie }}{\text { Population totale }} ;$

Population desservie $=$ Nombre de point d' eau $\times 250$
}

\section{Cadre d'étude}

Situé entre $6^{\circ} 57^{\prime}$ et $7^{\circ} 11^{\prime}$ de latitude nord et $2^{\circ} 31^{\prime}$ et $2^{\circ} 45^{\prime}$ de longitude est, le Hollidjé représente 4,6\% du territoire du département du Plateau avec une superficie de $150 \mathrm{~km}^{2}$ (Lanokou, 2015). Il est limité à l'est par une ligne nord-sud passant par les localités d'Igana et Akpatè et servant de séparation entre les pays Holli et Nago de Pobè. La dépression est limitée au nord par les pentes sud du revers du plateau de Kétou. Le secteur d'étude est limité à l'ouest par la commune, de Ouinhi et au sud par la ville de Pobè située sur une montée.

La figure 2 suivante présente la situation géographique du Hollidjé. 


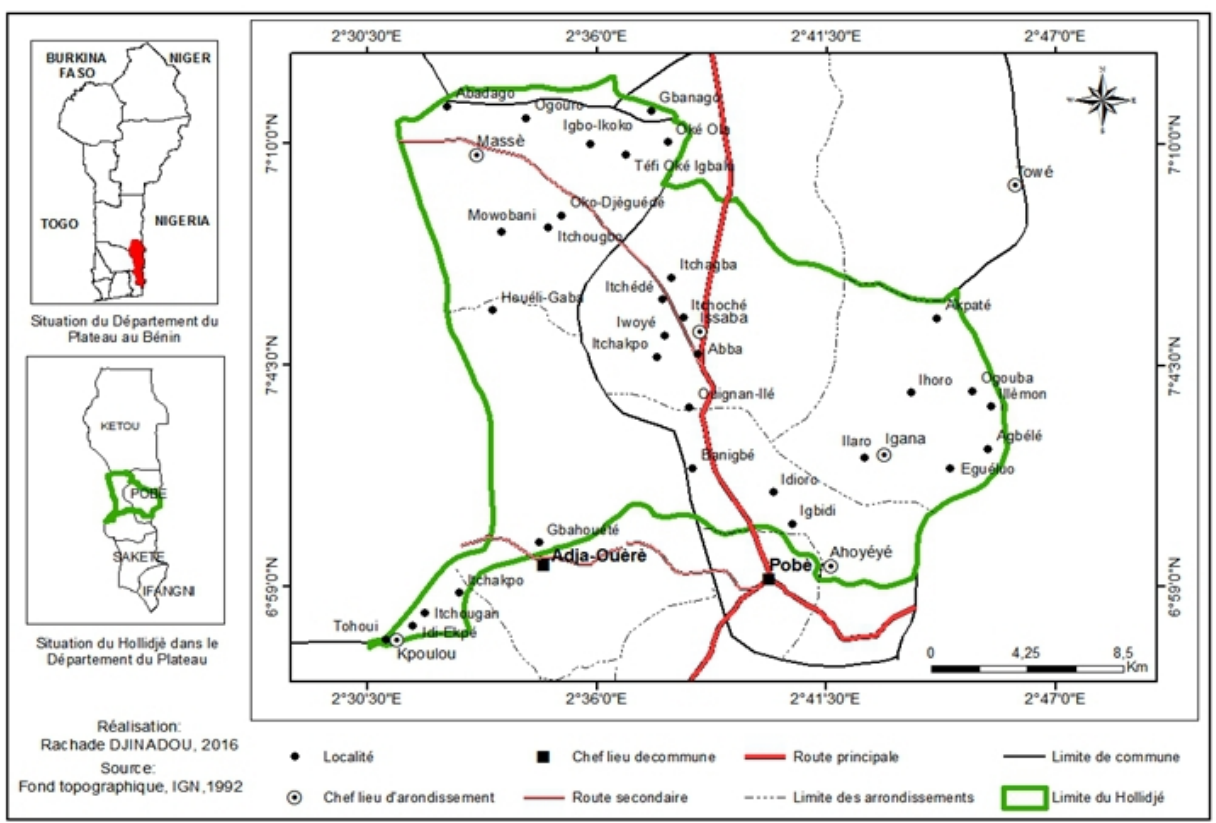

Figure 2: Situation géographique du Hollidjé

L'analyse de la figure 2 montre que le Hollidjé s'étend donc sur deux (2) communes, sept (7) arrondissements et trente-trois (33) villages. Sur le plan topographique, le Hollidjé est situé dans une zone de dépression avec une altitude inférieure à $50 \mathrm{~m}$. Il jouit d'un climat de type subéquatorial marqué par l'alternance de deux saisons pluvieuses (avril à juillet et septembre à novembre) et deux saisons sèches (novembre à mars et juillet à août). Le réseau hydrographique y est presque nul.

\section{Résultats et discussions}

Cette partie aborde à la fois les résultats obtenus et la discussion de ces derniers avec les autres études.

\section{Résultats}

Les résultats présentent les facteurs de la dynamique de population dans le Hollidje, la disponibilité et les besoins en eau dans le Hollidjé

\section{Dynamique de population dans le Hollidjé}

Les populations Holli du département du Plateau (Sud-est du Benin) se retrouvent à cheval entre les Communes de Pobè et d'Adja-Ouèrè. Ces peuples ruraux, représentent le tiers de la population de ces entités administratives avec une densité de l'ordre de 200 habitants $/ \mathrm{km}^{2}$ et une population croissante. Ainsi, entre les quatre recensements (c'est-à-dire 1979-1992-2002 et 2013), la population Holli est passée de 27894 habitants 
à 35445 habitants puis à 50847 habitants avant de passer à 69974 habitants soit un accroissement annuel moyen de l'ordre de 6,6 \% alors qu'il est de 3,5 $\%$ sur le plan national. La figure 2 présente l'évolution de la population Holli de 1979 à 2013.

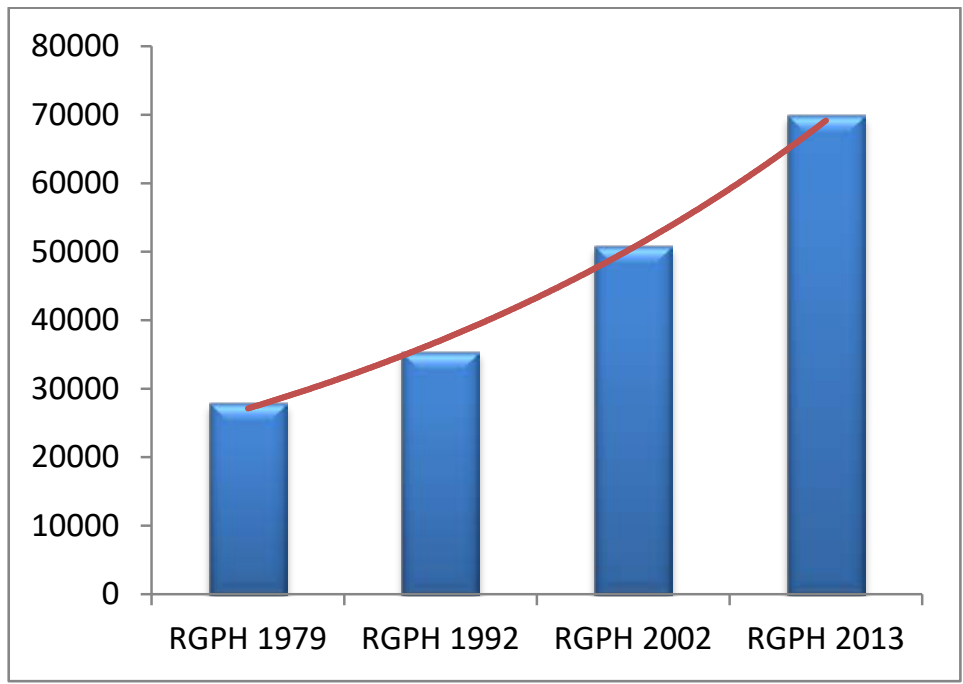

Figure 2 : Evolution de la population de 1979 à 2013 Source : INSAE 2016

Cette croissance est inégalement répartie selon les localités. La figure 3 présente la répartition spatiale des populations Holli entre 1979 et 2013.
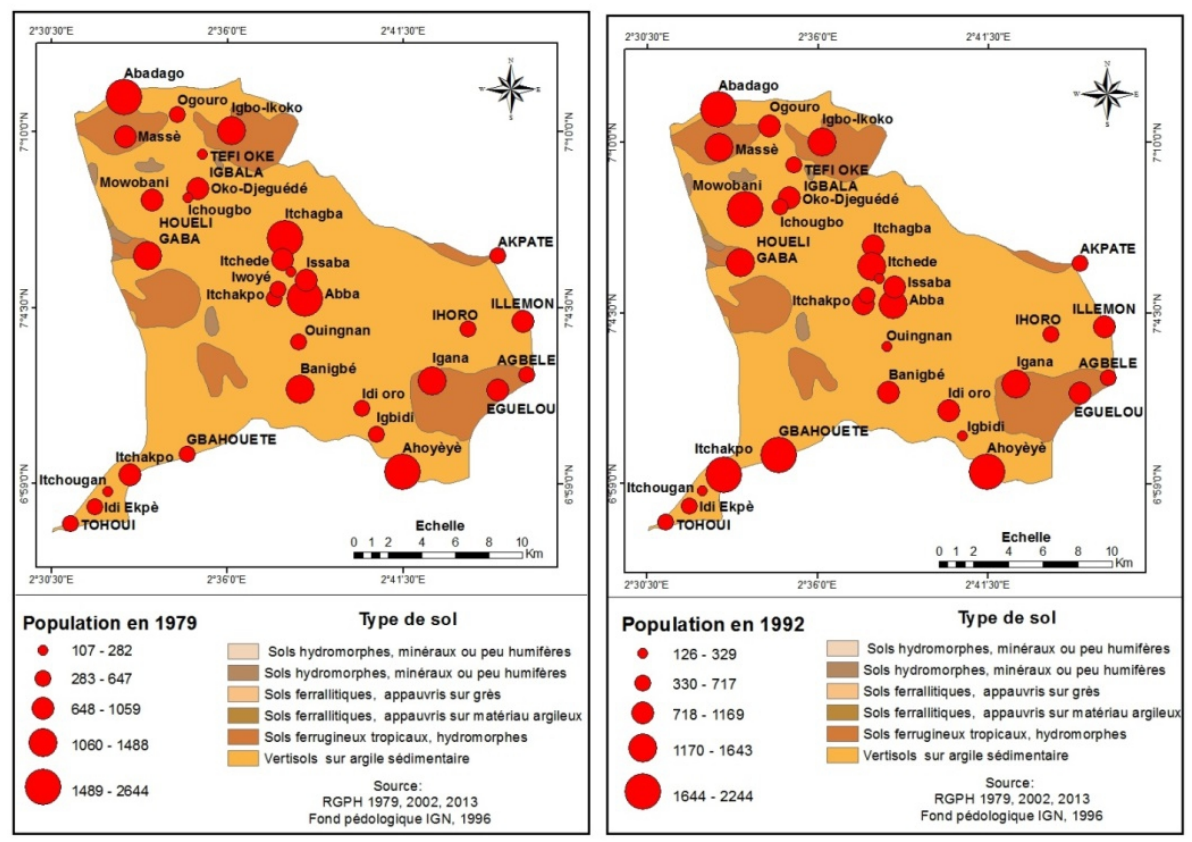

Figure 3 : Répartition spatiale de la population en 1979 et en 1992 Source : RGPH 1979 ; 1992 
Entre 1979 et 1992, la population holli a connu une augmentation de 7551 habitants, soit un accroissement de $27,07 \%$ en 13ans et un taux d'accroissement annuel de 2,08 \%. Cette evolution de la population peut être répartie en trois classes. La premiere est celle des localités ayant connu un fort taux d'accroissement et dont l'effectif de la population a été multiplié par deux ou parfois par trois. C'est le cas des villages: Abadago, Masse, Ogouro, Mowobani dans l'arrondissement de Masse. Il en est de meme pour villages d'Issaba, Itchakpo, Itchede et Iwoye dans l'arrondissement de Issaba ; des villages Houeligaba et Gbahouete dans l'arrondissement d'AdjaOuéré et des villages Itchakpo, Itchougan etIdi-Ekpe dans l'arrondissement de Kpoulou. La deuxieme classe est celle des localites qui ont connu un accroissement faible, c'est le cas des villages: Igana, Agbele, Eguelou, Akpate, Illemon, Ihoro dans l'arrondissement d'Igana. La troisieme classe est celle des localites ayant connu un accroissement negatif avec des effectifs des populations ayant diminue, c'est le cas des de Abba, Ouignan dans l'arrondissement de Issaba et des villages d'Ahoyeye, Idi-Oro, Igbi, Banigbe dans l'arrondissement de Ahoyeye.

La figure suivante presente le situation entre 2002 et 2013.

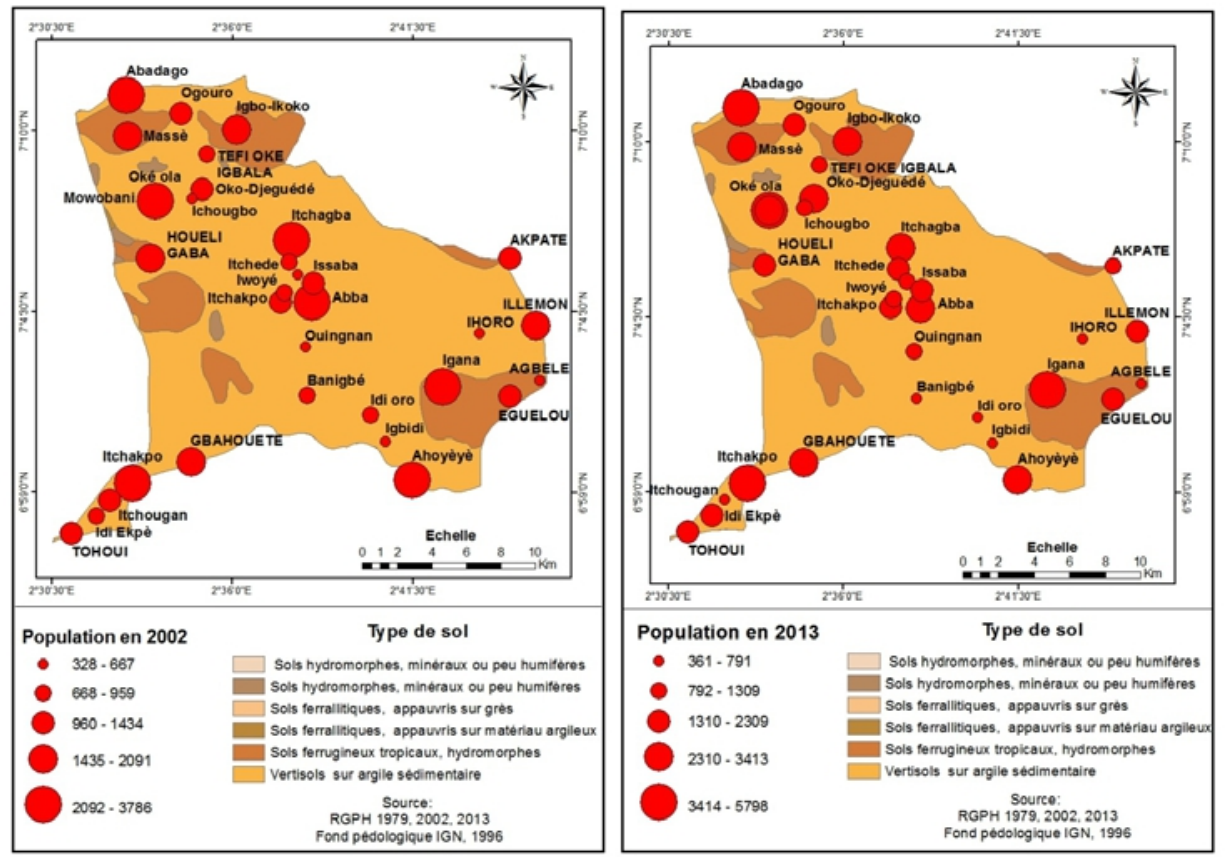

Figure 4 : Répartition spatiale de la population en 2002 et 2013

Source : RGPH 2002 ; 2013

Entre 1992 et 2002, cette croissance a été multipliée par deux, passant de 2,03 \% à 4,34 \% l'an mais avec les mêmes disparités spatiales. Cependant, entre 2002 et 2013, le taux de croissance est passée à 3,41 \% 
l'an, avec une croissance continue dans toutes les localités comme le montre la figure 4 . Cet accroissement rapide de la population Holli est la cause du fort taux de natalité dans la région comme la présente la pyramide des âges en 2013 de la figure 5.

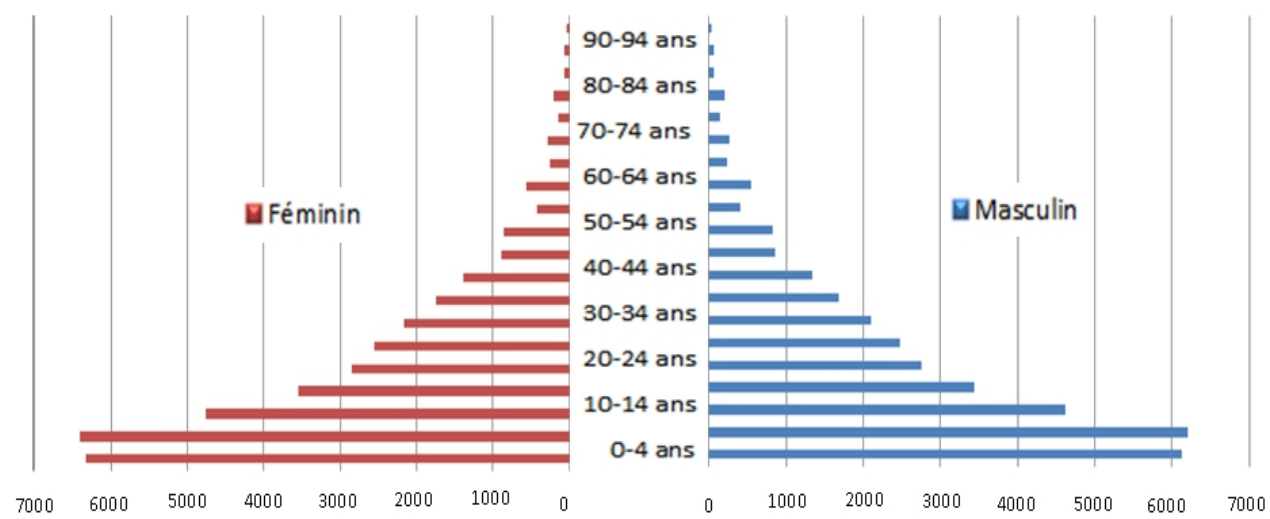

Figure 5 : Pyramide des âges de la population Holli en 2013 Source : RGPH 2013

Avec un taux de masculinité de 49,20\%, le Hollidjé est composé à $48,8 \%$, soit près de la moitié, des enfants dont l'âge est compris entre 0 et 14 ans. Ceci montre que la forte natalité est le facteur déterminant de l'accroissement démographique de ces populations.

Cet accroissement continu de population dans le Hollidjé entraine une forte demande en ressources naturelles et plus précisément l'accès à l'eau.

\section{Disponibilité et besoins en eau dans le Hollidjé}

La nature géologique et pédologique du Hollidjé ne permet pas la réalisation des infrastructures surtout celles liées à l'eau. Ainsi, en 2015 à l'échéance des Objectifs du Millénaire pour le Développement (OMD), l'état des lieux de l'accès à l'eau est présenté par la figure 6. 

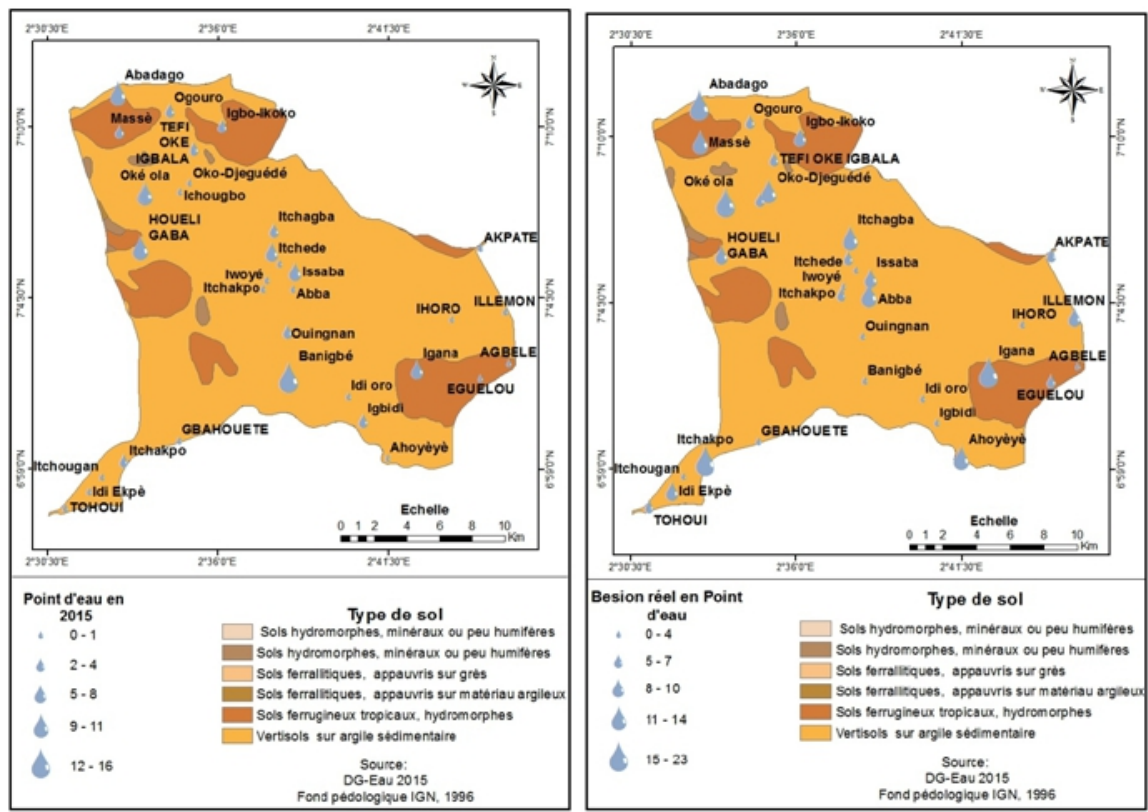

Figure 6 : Point d'eau existant et besoins d'eau dans le Hollidjé en 2015 Source : DG-Eau, 2015

En 2015, seuls 134 points d'eau étaient installés dans la région pour une population de 69974, soit 33500 habitants desservis et un taux de desserte de 47,87 \%. Cependant, du point de vue spatial, l'accès à l'eau est un véritable problème dans le Hollidjé. Ainsi, 07 localités (Itchakpo, Iwoye, Idi-Oro, Oko-djeguede, Gbahouete et Agbele et Tohoui) soit 10944 habitants et $15 \%$ de la population n'ont accès à aucune source d'eau potable soit un taux de desserte de $0 \%$. Dix (10) localités (Abba, Itchougbo, Ahoyeye, Akpate, Eguelou, Ilemon, Ihoro, Idi-Ekpe, Itchougan et Itchoche) soit une population de 16133 habitants et 23,05 \% de la population totale dispose seulement d'un point d'eau soit un taux moyen de desserte d'environ $10 \%$. Au total, près de $40 \%$ de la population Holli n'ont pas accès à une source d'eau ou ont un taux de desserte moins de $10 \%$.

En tenant compte de la population actuelle, les besoins étaient estimés à 280 points d'eau dans toute la région, avec douze localités ( Illemon, Masse, Igana, Agbadago, Ahoyeye, Itchagba, etc.) dont les besoins varient entre 10 et $24 \%$.

\section{Estimation de la population et des besoins en eau dans le Hollidjé à l'horizon 2030.}

A l'horizon 2030, la population Holli serait de 124788 habitants, avec des localités comme Igana ayant un très fort taux de croissance qui passera à 12124 habitants, alors que les localités à faible taux de 
croissance comme Idi-Oro et Banigbé seront respectivement de 707 habitants et 616 habitants. Du coup les besoins en eau potable vont aussi s'accroître et passeront de 280 en 2015 à 500 points d'eau potable à réaliser en 2030. Ainsi pour les localités d'Igana, Agbadago, KpoulouItchakpo, etc., il faut en moyenne 50 points d'eau. Les localités à croissance faible ont besoin d'une moyenne de 5 points d'eau (Ihoro, Banigbe, Itchougbo, Iwoye, Igbidi etc.) comme le montre la figure 7.
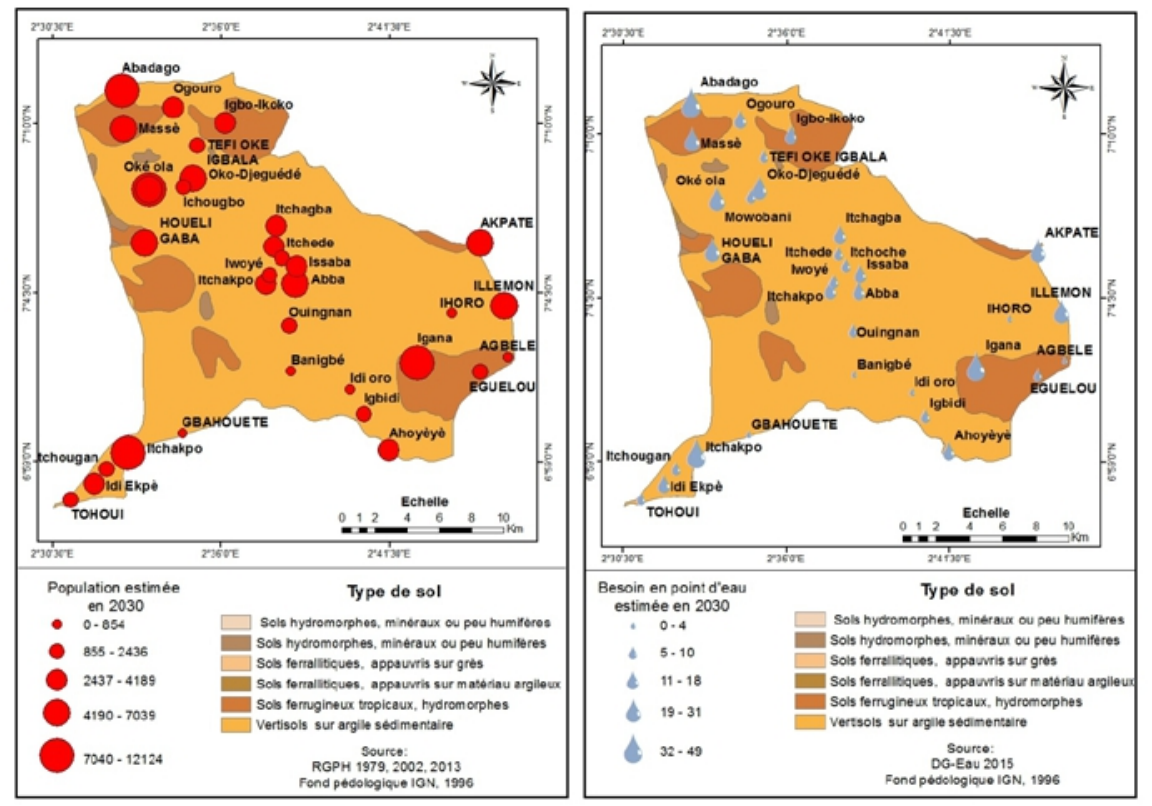

Figure 7 : Répartition spatiale de la population et des besoins en eau potable dans le Hollidjé à l'horizon 2030.

Source : DG-Eau, 2015

\section{Discussion}

La dynamique de la population mondiale est dû à la fois à la croissance du solde naturel et du solde migratoire. En Afrique subsaharienne, la forte natalité reste encore le principal facteur de l'augmentation démographique surtout dans les milieux ruraux (ONUFNUAP, 2014). Ces résultats confirment la tendance observée dans le Hollidjé avec une population composée a près de $50 \%$ de la tranche d'âge de 0-14 ans et une taille moyenne des ménages qui est d'environ 7 personnes.

Selon Lacoste (2003), la croissance démographique rapide permet à l'eau d'acquérir une valeur dont le cours est indexé sur la pénurie annoncée et lié à la consommation individuelle en pleine explosion. Ainsi, les besoins en eau de la population Holli est en nette augmentation. Evaluée à 280 points d'eau en 2015 dans la perspective d'atteindre l'OMD 7 qui est de réduire de 
moitié le nombre de personnes non desservie en eau potable et assainissement, la couverture en points d'eau n'était que 134, soit un taux de desserte de 47,87 \% avec de grandes disparités spatiales. En effet, plus 55 \% de la population ne sont pas desservis en source d'eau améliorée ou sont faiblement desservis. Parmi ceux-ci $15 \%$ n’ont accès à aucune source d'eau potable et $23 \%$ sont desservis à hauteur de moins de $10 \%$. Ainsi, ces populations ont alors recours à des eaux impropres à la consommation et sont exposées à de nombreuses maladies hydriques (Lagnika et al., 2014). Ces résultats sont confirmés par de nombreuses études antérieures. Ainsi pour l'OMS-UNICEF (2010), l'Afrique sub-saharienne en 2015 ne pourra pas atteindre l'OMD lié à l’eau et assainissement. Malgré les avancées réalisées, l'effectif des personnes non desservies en eau potable va augmenter de 47 millions avec les 3/4 dans les zones rurales. Pour Babadjidé (2011), les eaux de boisson des populations rurales de Kétou et Pobè sont en général contaminées par des microbes issus des matières fécales qui sont déposées aux alentours des sources d'eau, faute de latrines.

En raison de la non atteinte des OMD en 2015, les Nations Unies ont prolongé l’échéance jusqu'en 2030 et redéfini les objectifs pour un développement durable. Dans le secteur de l'eau et assainissement, l'ODD 6 vise à : " Garantir l'accès de tous à l'eau et à l'assainissement et assurer une gestion durable des ressources en eau » (UNICEF, 2015 et https://www.eda.admin.ch/post2015/fr).

Face à l'ampleur de la croissance démographique et aux caractéristiques géo-pédologiques du Hollidjé qui ne sont pas du tout favorable à l'installation des infrastructures d'approvisionnement en eau potable et assainissement (Lanokou, 2016), l'objectif 6 des ODD et surtout le premier point qui consiste à «d'ici à 2030, assurer l'accès universel et équitable à l'eau potable, à un coût abordable » ne sera pas atteint. En effet, en 2030, l'effectif de la population Holli va doubler (69974 hbts à 124788 hbts) et les besoins en points d'eau vont aussi s'accroitre pour passer à 500. Mais avec les tendances actuelles, où des localités entières ne disposent du tout pas de source d'eau potable, il va falloir définir de nouvelles stratégies pour y parvenir.

\section{Conclusion}

La population Holli du sud-est du Benin, connait un accroissement au-dessus du niveau national. Cette population n'a pas accès à une source d’eau améliorée dans son ensemble et les facteurs géophysiques de ce milieu ne favorisent pas l'installation d'infrastructures d'assainissement. Malgré les efforts fournis, l’objectif du millénaire lié à l'eau n’a pas été atteint en 2015 et si la tendance reste ainsi, celui du développement durable risque de ne pas l'être aussi à l'horizon 2030. 
Si l'on veut atteindre la cible relative à l'eau potable et à l'assainissement dans la région, il est indispensable que tous les acteurs concernés intensifient leur action de manière concertée.

Ainsi, le pays doit définir des objectifs à atteindre, élaborer des plans d'action réalistes et à prévoir les ressources financières et humaines nécessaires pour garantir à cette population un accès durable à l'eau potable et à l'assainissement de base, tout en tenant compte des besoins fondamentaux des plus vulnérables et des plus démunis. Cet effort doit être entrepris non seulement pour des raisons humanitaires, mais aussi parce qu'il est rentable et permet de réduire considérablement les coûts de la santé, et qu'il influe directement sur les trois facteurs qui constituent le fondement de la lutte contre la pauvreté: la santé, l’équité et la croissance économique. Co

\section{References:}

1. Azontonde A., 1991: Propriétés physiques et hydrauliques des sols au Bénin, CENAP, Abomey-Calavi, $50 \mathrm{p}$.

2. Babadjidé C. L. et Vissin E. W., 2011: Qualité d'eau de consommation et les maladies hydriques dans la commune de Kétou (Benin), XXVIe Colloque de l'Association Internationale de Climatologie, pp 81-86.

3. Dansou B. S. et Odoulami L., 2013: Impacts socio-économiques des inondations dans la dépression d'Issaba (Commune de Pobè au Benin). Revue de géographie de l'Université de Ouagadougou, Numéro 002, septembre 2013, pp 157-171.

4. Dansou B. S. et Odoulami L., 2012: Problématique des inondations dans la commune de Pobè, in Mélange MAC, DGAT/FLASH/UAC, mars 2012, pp. 515-525.

5. Djinadou, R. O.A., 2012: Dynamique urbaine et problèmes sociosanitaires dans la ville de Cotonou, mémoire de DEA, UAC, EDP, FLASH, $72 \mathrm{p}$.

6. Djinadou R. O. A. et Kouchadé A. C., 2015: Approche genre dans la perception des populations Holli de la gestion participative des forets et plantations de la Lama (Benin), Cahiers du CBRST, Cotonou (Benin), ISSN 1840-703X, Numéro 7, vol 02, pp 117-134

7. INSAE, 2016: Effectifs de la population des villages et Quartiers de ville du Benin (RGPH-4 2013), Cotonou, 85 p.

8. Lagnika M., 2014: Caractéristiques physico-chimiques de l'eau de puits dans la commune de Pobè (Benin), Journal of Applied Biosciences, ISSN-1997-5902, pp 6887-6897.

9. Lanokou C.M., 2016: Extrêmes climatiques et mise en valeur agricole des terres noires dans la dépression médiane au sud-Benin, Thèse de doctorat de l’EDP, UAC, 313 p. 
10. Niang C.I., 2008: Sante, société et politique en Afrique, Livre vert du CODESRIA, Dakar, ISBN-978-2-86978-22-8, 48 p.

11. OMS-UNICEF, 2010: Atteindre l'OMD relatif à l'eau potable et assainissement: Le Défi urbain et rural de la décennie, Rapport, ISBN-97892-4-2563252, New York, 48 p.

12. ONU, 2015: Les objectifs de développement durable, Recherche en ligne, 29 septembre 2016. https://www.eda.admin.ch/post2015/fr.

13. ONU-FNUAP, 2014: Atteindre l'OMD relatif à l'eau potable et à l'assainissement: Bilan de la situation au niveau mondial et régional en 1990 et 2004, et efforts à entreprendre au cours de la période 2005-2015, Rapport, New York, 43 p.

14. UNICEF, 2015: Eau potable et Assainissement : Disparité entre zone rurale et urbaine, Rapport, New York, 32 p. 